\title{
STELLAR ROTATION IN OPEN CLUSTERS
}

\section{(Review Paper)}

\author{
HELMUT A. ABT \\ Kitt Peak National Observatory*, Tucson, Ariz., U.S.A.
}

\begin{abstract}
The characteristics of rotational velocities in fifteen open clusters and associations are summarized. The differences in mean rotational velocities observed between individual groups are found to be due primarily to three mechanisms: evolutionary expansion, tidal coupling of rotational and orbital motion in binaries, and magnetic braking in Ap stars.
\end{abstract}

\section{Introduction: The Problem}

Recent work on stellar rotation in open clusters and associations has been motivated primarily by the following questions:

(1) Are the stellar rotational velocities in various clusters the same or different for each group, and

(2) If different, what causes the differences?

This paper is confined entirely to a discussion of answers to these questions.

As was true for so many spectroscopic problems, the first results came from Struve (1945), who pointed out that the Pleiades and Hyades have very different mean rotational velocities as a function of spectral type than do field stars. The brighter stars in the Pleiades and Hyades have unusually high and low mean rotational velocities, respectively, although Anderson et al. (1966) and Kraft (1965) showed that the fainter stars in these clusters have the same mean rotational velocities as the field stars. An example of the differences between clusters is shown in Figure 1. It seems apparent that clusters often do differ in their mean projected rotational velocities, so the remainder of this paper will deal with possible explanations for such differences.

The question immediately arises whether the mean measured values of $V \sin i$ are unusual because of high or low equatorial rotational velocities, $V$, or because of preferential inclinations, $i$, of rotational axes. It seems very likely that the former case occurs for the following reasons, which are partly due to Kraft (1965):

(1) Clusters, such as the Hyades and Coma, that have many characteristics in common also share an unusual dependence of $\langle V \sin i\rangle$ on spectral type. Since the directions of these two clusters from the solar system are very different $\left(b^{\mathrm{II}}=-24^{\circ}\right.$ and $+84^{\circ}$, respectively), it would be surprising if their $V$ 's and $i$ 's combined to give the same values of $\langle V \sin i\rangle$.

(2) Investigation (Kraft, 1965) of the inclinations of orbital planes of visual binaries in these two clusters showed no preferential orientation, and one might expect the rotational and orbital axes to be roughly aligned.

* Operated by the Association of Universities for Research in Astronomy, Inc., under contract with the National Science Foundation. 
(3) It would be surprising if the B stars in a cluster showed one preferential orientation and the A stars a different preferential orientation.

(4) The difference between the $\langle V \sin i\rangle$ for field stars and for the brightest stars in the $\alpha$ Persei cluster, for example, is larger than would occur for random orientation of axes (for the field stars) and complete alignment seen equator-on (for the cluster).

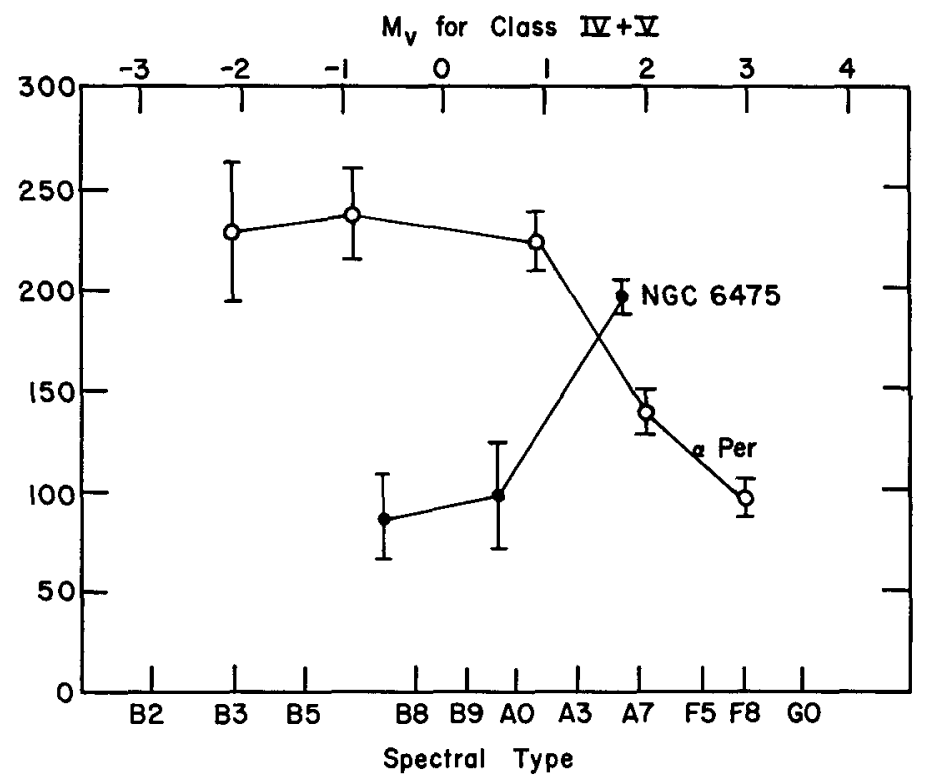

Fig. 1. Mean rotational velocities in two open clusters. The stars have been grouped by absolute magnitude; the corresponding spectral types, using Blaauw's (1963) calibration, are given. Half the length of the vertical bars is the probable error in the mean value of $V \sin i$ for each group.

(5) Huang and Wade (1966) showed that the orbital planes of field eclipsing binaries show no preferential galactic orientation; in closely-spaced binary systems we would expect rough alignment of rotational and orbital axes, so the lack of a preferential galactic orientation should also apply to the rotational axes of field stars.

Therefore we shall assume in the following discussion that alignment of axes does not contribute appreciably to the unusual projected rotational velocities that are observed.

\section{Cluster Data and Discussion}

The first compilations of data from various clusters and associations were by Treanor (1960) and Abt and Hunter (1962), although most of the groups discussed by them have been studied more thoroughly and more carefully since then. Table I gives references to the most recent studies involving appreciable numbers of stars per cluster.

The results from these fifteen groups can be summarized as follows:

(1) Relative to field stars, most clusters show unusually large or unusually small 
TABLE I

References to rotational velocity studies

\begin{tabular}{llc}
\hline $\begin{array}{l}\text { Cluster or } \\
\text { association }\end{array}$ & Reference & $\begin{array}{l}\text { No. of } \\
\text { stars }\end{array}$ \\
\hline a Persei & Kraft (1967a) & 73 \\
Coma & Kraft (1965) & 30 \\
h and $\chi$ Persei & Slettebak (1968b) & 83 \\
Hyades & Kraft (1965) & 78 \\
IC 4665 & Abt and Chaffee (1967) & 27 \\
M39 & Meadows (1961) & 18 \\
NGC 2516 & Abt et al. (1969) & 30 \\
NGC 6475 & Abt and Jewsbury (1969) & 27 \\
Pleiades & Anderson et al. (1966) & 57 \\
Praesepe & Dickens et al. (1968) & 56 \\
Ursa Major & Geary (1969) & 48 \\
I Lacerta & Abt and Hunter (1962) & 26 \\
I Orion & McNamara and Larsson (1962), McNamara (1963) & 83 \\
II Perseus & Treanor (1960) & 10 \\
Sco-Cen & Slettebak (1968a) & 82 \\
\hline
\end{tabular}

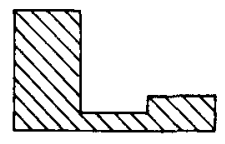

NGC 6475
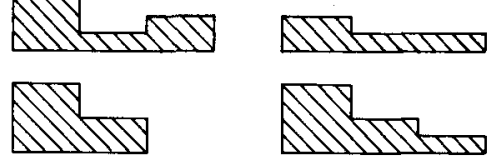

IC 4665

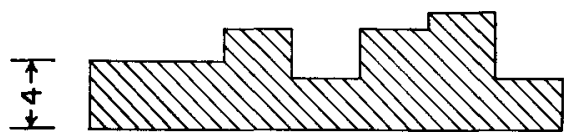

I Ori

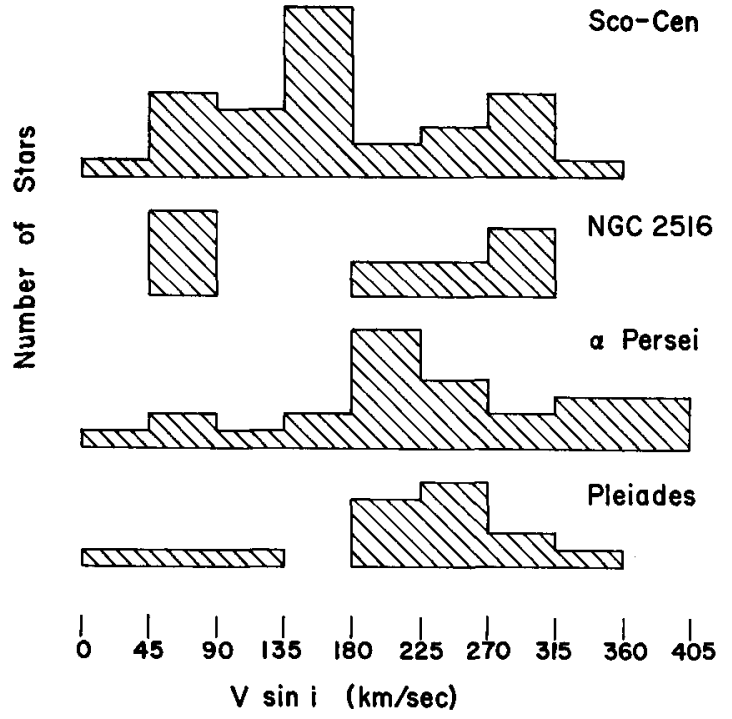

Fig. 2. Frequencies of values of $V \sin i$ in seven clusters and associations. All Ap stars have been excluded. 
mean rotational velocities for their brighter stars, although their fainter stars tend to conform to the field-star relation.

(2) In many clusters there is a bimodal distribution of values of $V \sin i$ such that there are very few stars of intermediate $(100<V \sin i<200 \mathrm{~km} / \mathrm{sec})$ line broadening (Figure 2).

(3) In some clusters, such as the Pleiades (Struve, 1945), Sco-Cen (Slettebak, 1968a) and NGC 2516 (Abt et al., 1969), there is a tendency for the rapid rotators to concentrate to the projected center of the cluster, but this concentration is not apparent for other groups, such as $h$ and $\chi$ Persei (Slettebak, 1968b).

Before attempting to interpret some of these characteristics, let us summarize the data on field stars. It is a remarkable tribute to our host that his initial papers (Slettebak, 1949, 1954, 1955; Slettebak and Howard, 1955) after 15-20 years still provide the best fundamental data on stellar rotational velocities; very few of his results require revision because of line doubling, photometric errors, or errors of analysis. His distribution of $\langle V \sin i\rangle$ with spectral type is shown in Figure 3 for 279 stars of

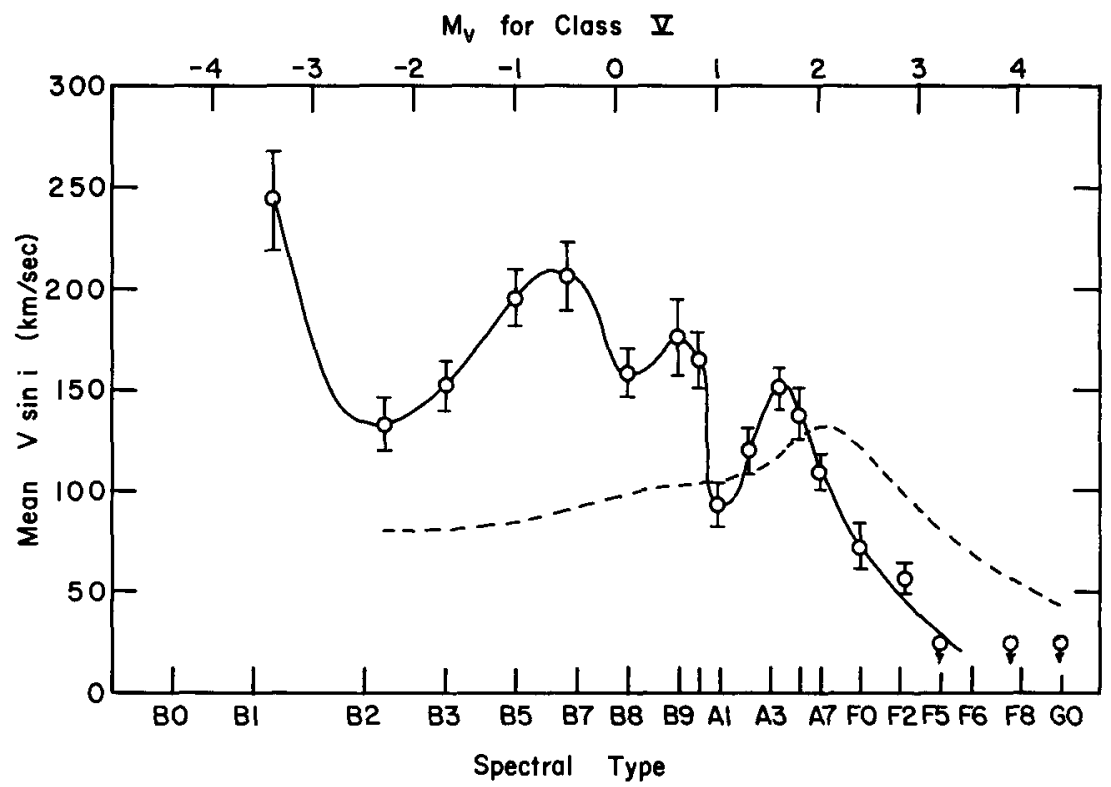

Fig. 3. The distribution of $\langle V \sin i\rangle$ with spectral type for stars of luminosity class $V$ (individual means with probable error bars, and the solid line) and class III (dotted line), as derived by Slettebak and Howard. The calibration of type vs. absolute magnitude is taken from Blaauw (1963). Note the minima at $\mathrm{B} 2, \mathrm{~A} 1$, and perhaps $\mathrm{B} 8$ for the class $V$ stars.

luminosity class $\mathrm{V}$ and 56 stars of class III. The curve for class V stars shows the marked decrease at about F2 where the outer convective zone commences, an uncertainty among the O-type and early B-type stars where turbulence contributes appreciably but to an unknown amount to the line broadening, and various maxima and minima among the B's and A's. 
The maxima and minima, originally pointed out by Boyarchuk and Kopylov (1958) from less homogeneous material, have been discussed by Abt and Hunter (1962), who suggested that appreciable contributions to the sample of field stars by individual clusters and associations having abnormally high or low rotational velocities at some spectral types may be distorting the mean curve. For instance, removal of the 21 Cassiopeia-Taurus B1-B3 stars from the sample of 66 such field stars will partly eliminate the minimum at B2. Inclusion of the extensive material on A stars by Palmer et al. (1968) makes this explanation less likely (see the discussion at the end of this paper).

With regard to the causes of differences between clusters, we can think of three likely explanations, which will be discussed in the following three sections.

\section{A. EVOLUTIONARY EXPANSION}

As the brighter stars in a cluster leave the zero-age main sequence and expand, their rotational velocities decrease at a rate that is inversely proportional to the radius if the outer layers retain their original angular momenta, or at a slower rate if angular momentum is transferred within the stars such that they always rotate as rigid bodies.

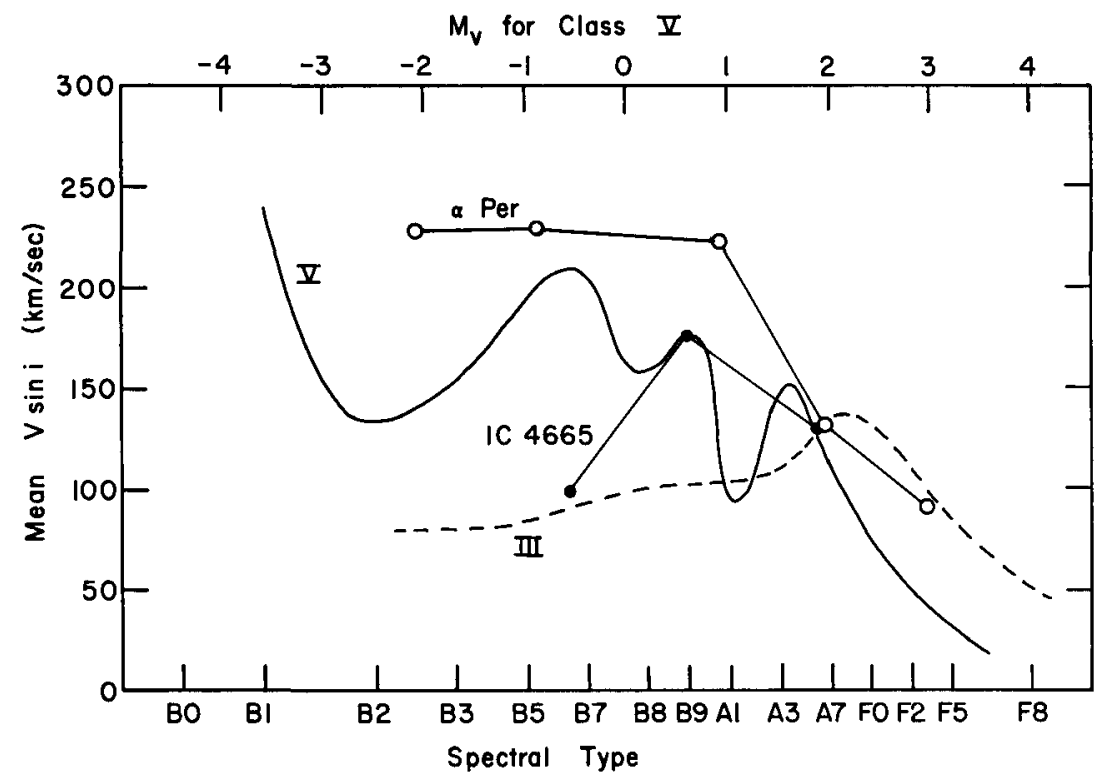

Fig. 4. Dependence of mean rotational velocities on spectral type in the $\alpha$ Persei and IC 4665 clusters, compared with the relation for field stars of luminosity classes $\mathrm{V}$ and III.

A third possibility is that of differential rotation involving a rapidly rotating core and slowly rotating envelope (Dicke, 1964), or the inverse. Observationally, the rotational velocities of the brightest stars in clusters should approach those of class III field stars. In Figure 4 we see a cluster (IC 4665) in which such an evolutionary expansion would 
seem to explain the low rotational velocities of the brightest stars. Unfortunately we do not yet have good spectral classifications for the stars in that cluster and some others (NGC 6475) showing that dip, to see whether most or all of their brightest stars are of luminosity class III. However, the brightest stars in IC 4665 have a maximum radial expansion from their zero-age sizes (Abt and Chaffee, 1967) of only $26 \%$, which is probably much too small to explain their 2-fold decrease in $V \sin i$ relative to field stars. What is even more convincing in demonstrating that evolutionary expansion is not the only cause of abnormal rotational velocities in clusters is the case of the $\alpha$ Persei cluster, where the evolved stars have larger, rather than smaller, mean rotational velocities than field stars.

A group in which the brighter stars have abnormally low rotational velocities and in which good MK types are available by Morgan (Morgan et al., 1955), Slettebak and Howard (1955), or Sharpless (1952) is the I Orion association. In Figure 5 we show the

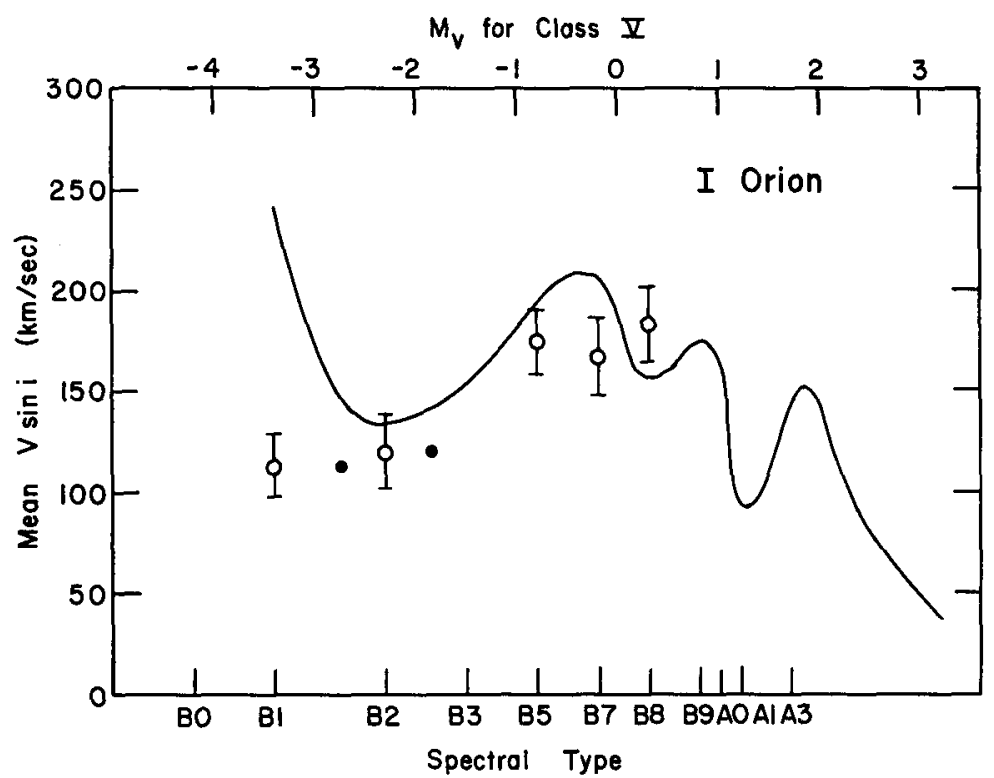

Fig. 5. The mean rotational velocities (McNamara and Larsson, 1962) of 35 bright I Orion stars (left two circles) with MK types indicating luminosity class V, and 33 fainter stars (right three circles) assumed to be of class V (McNamara, 1963) are plotted according to their mean spectral types. The solid line is for class $\mathrm{V}$ field stars. If the brighter Orion stars are plotted according to their absolute magnitudes (black dots), the discrepancy with the field stars is much less.

mean rotational velocities for class $\mathrm{V}$ stars in I Orion relative to the class $\mathrm{V}$ field star relation. The fainter stars in the association are approximately normal (meaning like field stars), but the B0-B1.5 stars rotate too slowly. In this case the inverse evolutionary effect may be present: the class $V$ association stars may be less evolved than the average class $\mathrm{V}$ field star. The black dots in Figure 5 show to where the left two means are 
shifted if those points are plotted by absolute magnitude, using an apparent distance modulus of $8.5 \mathrm{mag}$, rather than by spectral type. In this case the discrepancy with field stars is less marked.

We conclude that the evolutionary expansion and consequent effect on the rotational velocities of stars should be considered, but that this effect alone cannot explain many of the differences between individual clusters and field stars.

\section{B. TIDAL COUPLING IN BINARIES}

There are at least two ways in which the initial rotational velocities of stars may be gradually modified: by tidal interaction in closely-spaced binaries and by magnetic braking in magnetic stars. The latter effect will be discussed in the next section. If some clusters differ from each other or from field stars in their numbers of spectro-

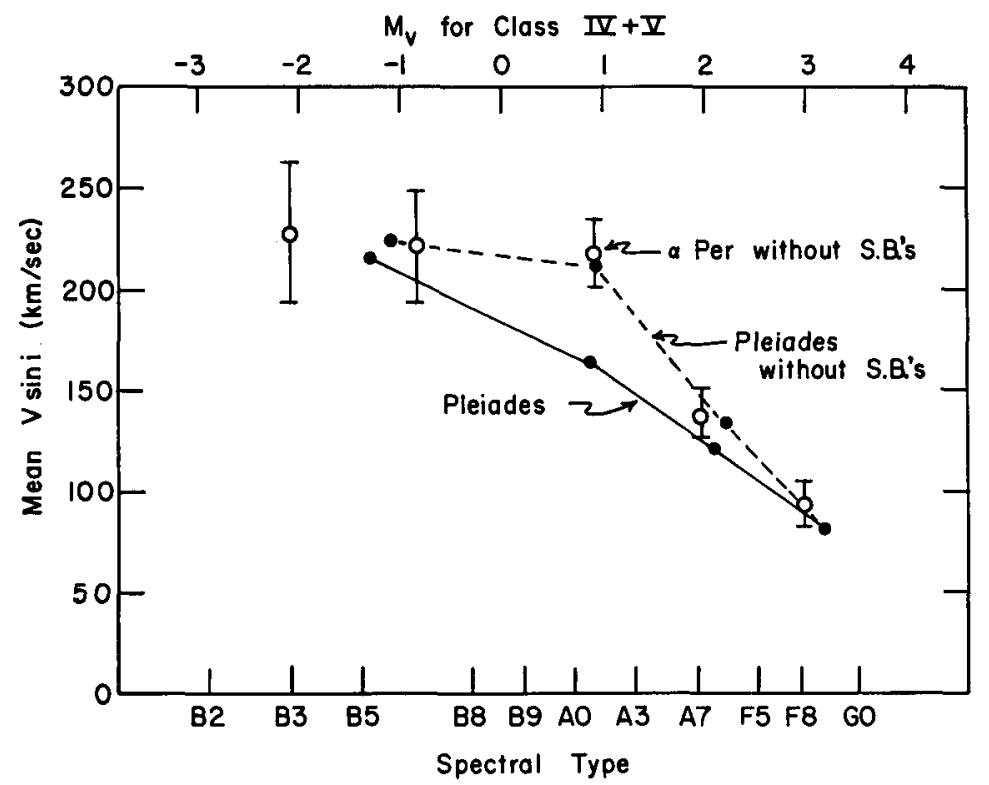

Fig. 6. Mean rotational velocities for all the Pleiades stars (solid line) and excluding the spectroscopic binaries (dashed line) compared with similar quantities for the $\alpha$ Persei cluster (circles with error bars), excluding binaries.

scopic binaries, we might expect that their modified mean rotational velocities would differ.

An interesting test case is that of the Pleiades and $\alpha$ Persei clusters. Eggen (1965) suggested that since these two clusters, and several others, have common space motions and similar color-magnitude diagrams, they have a common origin. Kraft (1967a) inquired whether their rotational velocity distributions are similar. Those distributions are different if all their stars are included. However, although both clusters have few spectroscopic binaries, the Pleiades has more than the $\alpha$ Persei cluster 
among the early A-type stars, where the rotational velocity distributions depart significantly. After excluding all definite spectroscopic binaries (and the few Am and Ap stars) in both groups, the rotational velocity distributions were found to be similar (Figure 6).

It is now apparent that some clusters, such as the $\alpha$ Persei cluster (Heard and Petrie, 1967) and Pleiades (Abt et al., 1965) have very few spectroscopic binaries while others, such as NGC 6475 (Abt et al., 1970) have many. Also, where the frequency of binaries is low, the mean rotational velocities are high, and where the binaries are frequent, the mean rotational velocities are low.

We conclude that tidal interaction in closely-spaced binaries is effective in reducing rotational velocities, and that a large part of the differences between clusters in their mean rotational velocities is due to different binary frequencies. In a sense we have succeeded only in shifting the problem from trying to explain the various mean rotational velocities to trying to explain the different binary frequencies.

\section{MAGNETIC BRAKING}

The rotational velocities of Am (Slettebak, 1955; Abt, 1965) and Ap (Slettebak, 1955; Abt et al., 1967) stars are consistently low relative to normal stars. For the Am stars the slow rotation seems to be due to tidal coupling in binaries, which are very frequent among such stars. However, it seems likely that binaries are not frequent among Ap stars (Babcock, 1958) and that another mechanism must be found for producing the low rotational velocities in these stars. After consideration of the extremely large amounts of angular momentum available in gaseous nebulae, we do not think that single stars are likely to reach the main sequence with low rotational velocities. It seems more likely that magnetic braking, either within a star rotating differentially or between the stellar and interstellar magnetic fields, will cause the slow rotation.

A test case for this idea is that of NGC 2516 that, according to Eggen (1965), has a similar space motion and color-magnitude diagram to the Pleiades and $\alpha$ Persei cluster. Kraft found the latter two clusters to have similar rotational velocities, after allowance for differences in binary frequencies, so we are somewhat more confident about finding similar rotational velocities in NGC 2516. However, that cluster has many Ap stars (Abt and Morgan, 1969): seven of the 23 brightest main-sequence stars are definite or probable Ap stars. The binary frequency for this cluster is not yet known. With the Ap stars included, the rotational velocities are different than for the Pleiades (Figure 7), both with binaries included, but with the Ap stars excluded, the rotational velocities are similar.

We conclude in this section that different frequencies of Ap stars in clusters can help cause differences in mean rotational velocities.

\section{OTHER MECHANISMS}

Other mechanisms that may produce differences between clusters by modifying stellar rotational velocities are (1) differential rotation, either in the surface layers or with depth, (2) age effects, such as those demonstrated by Kraft (1967b) (this was suggested 


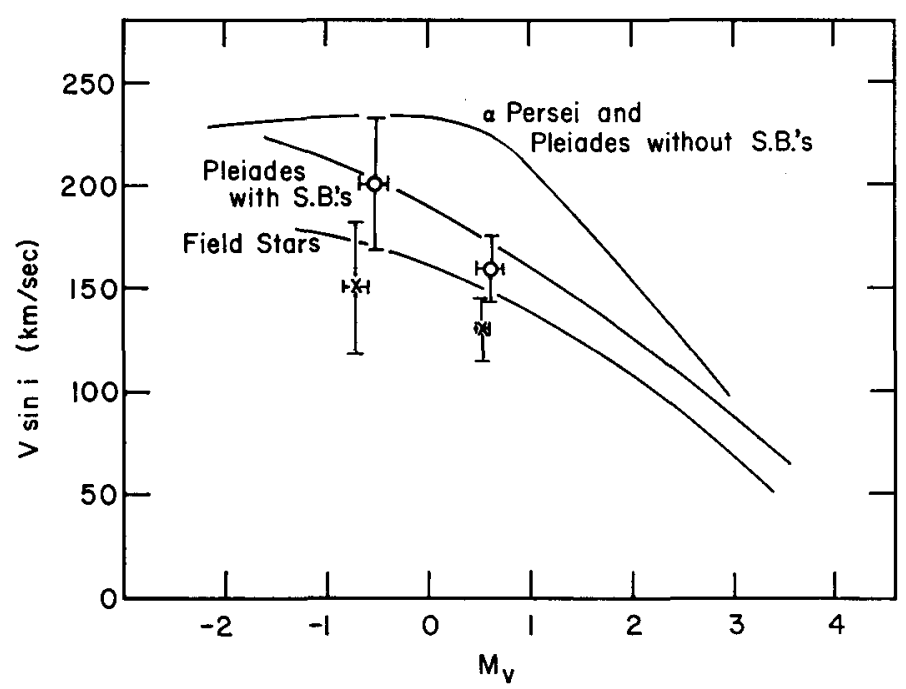

Fig. 7. Mean rotational velocities (x's) and probable error bars for 30 stars in NGC 2516. If the seven definite or likely Ap stars in this cluster are excluded, the resulting mean rotational velocities (circles) agree with the Pleiades relation, including spectroscopic binaries in both clusters (reprinted courtesy of the Astronomical Journal).

by Ostriker in the Colloquium discussion), (3) magnetic field effects, (4) mass loss, and (5) possible effects of the initial total cluster mass upon the cluster characteristics. For most of these it is not clear why stars of the same mass and age but in different clusters should produce differing results.

The goal for coming research is to see whether, after allowance for all the obvious effects (evolution, differing duplicity, differing frequencies of peculiar stars, etc.), the known clusters have a common dependence of mean rotational velocity on spectral type.

\section{References}

Abt, H. A.: 1965, Astrophys. J. Suppl. 11, 429 (No. 102).

Abt, H. A. and Chaffee, F. H.: 1967, Astrophys. J. 148, 459.

Abt, H. A. and Hunter, J. H., Jr.: 1962, Astrophys. J. 136, 381.

Abt, H. A. and Jewsbury, C. P.: 1969, Astrophys. J. 156, 983.

Abt, H. A. and Morgan, W. W.: 1969, Astron. J. 74, 813.

Abt, H. A., Barnes, R. C., Biggs, E. S., and Osmer, P. S.: 1965, Astrophys. J. 142, 1604.

Abt, H. A., Chaffee, F. H., and Suffolk, G.: 1967, Astron. J. 72, 783.

Abt, H. A., Clements, A. E., Doose, L. R., and Harris, D. H.: 1969, Astron. J. 74, 1153.

Abt, H. A., Levy, S. G., Baylor, L. A., Hayward, R. R, Jewsbury, C. P., and Snell, C. M.: 1970, Astron. J. 75, in press.

Anderson, C. M., Stoeckly, R., and Kraft, R. P.: 1966, Astrophys. J. 143, 299.

Babcock, H. W.: 1958, Astrophys. J. 128, 228.

Blaauw, A.: 1963, Basic Astronomical Data (ed. by K. Aa. Strand), University of Chicago Press, Chicage, Ill., p. 383.

Boyarchuk, A. A. and Kopylov, I. M.: 1958, Astron. Zh. 35, 804; trans.: 1959, Soviet Astron. $2,752$.

Dicke, R. H.: 1964, Nature 202, 432.

Dickens, R. J., Kraft, R. P., and Krzeminski, W.: 1968, Astron. J. 73, 6. 
Eggen, O. J.: 1965, Ann. Rev. Astron. Astrophys. 3, 235.

Geary, J. C.: 1969, in preparation.

Heard, J. F. and Petrie, R. M.: 1967, in Determination of Radial Velocities and Their Applications, I.A.U. Symposium No. 30, Academic Press, London, p. 179.

Huang, S.-S. and Wade, C., Jr.: 1966, Astrophys. J. 143, 146.

Kraft, R. P.: 1965, Astrophys. J. 142, 681.

Kraft, R. P.: 1967a, Astrophys. J. 148, 129.

Kraft, R. P.: 1967b, Astrophys. J. 150, 551.

McNamara, D. H.: 1963, Astrophys. J. 137, 316.

McNamara, D. H. and Larsson, H. J.: 1962, Astrophys. J. 135, 748.

Meadows, A. J.: 1961, Astrophys. J. 133, 907.

Morgan, W. W., Code, A. D., and Whitford, A. E.: 1955, Astrophys. J. Suppl. 2, 41.

Palmer, D. R., Walker, E. N., Jones, D. H. P., and Wallis, R. E.: 1968, Roy. Obs. Bull., No. 135.

Sharpless, S.: 1952, Astrophys. J. 116, 251.

Slettebak, A.: 1949, Astrophys. J. 110, 498.

Slettebak, A.: 1954, Astrophys. J. 119, 146.

Slettebak, A.: 1955, Astrophys. J. 121, 653.

Slettebak, A.: 1968a, Astrophys. J. 151, 1043.

Slettebak, A.: 1968b, Astrophys. J. 154, 933.

Slettebak, A. and Howard, R. F.: 1955, Astrophys. J. 121, 102.

Struve, O.: 1945, Pop. Astron. 53, 259.

Treanor, P. J., S.J.: 1960, Monthly Notices Roy. Astron. Soc. 121, 503.

\section{Discussion}

Jaschek: Are the periods of all Ap stars which are spectroscopic binaries equal to the periods of their magnetic variations? If not, would that not throw doubt upon the possibility that magnetism influences rotation?

$A b t$ : The only Ap star in a binary with identical magnetic and orbital periods is HD 98088 , which has identical binary, rotational, and magnetic periods. In long-period systems there is probably a tendency toward synchronism, but it need not be achieved.

Preston: The spectroscopic binary HR 710 seems to be an exception. The magnetic field does not vary periodically in the binary period of 3 days.

Rosendhal: If you add together the results for the individual clusters do you recover something which looks like the field star distribution?

$A b t$ : Yes, very closely.

Ostriker: Isn't it likely that, for the later spectral types, a fourth explanation (suggested by Kraft) must be considered? An age effect may exist with the older stars (at about the same spectral type) having the lower surface velocities.

$A b t$ : Yes, this may explain why the $\langle v \sin i\rangle$ for the later-type stars in a young cluster like the Pleiades is larger than in an older cluster like the Hyades and Coma.

Kraft: NGC 2516 is very remarkable because of the large number of Ap's. Even if the rectified rotation function (omitting Ap's) is the same as that of the Pleiades and $\alpha$ Per cluster, it does not support Eggen's suggestion of a common origin of these three clusters because one cannot explain why NGC 2516 has so many Ap's and the other two clusters have almost none.

$A b t$ : Yes. Perhaps all we can do is shift the problem from trying to explain different rotational velocities to trying to explain different frequencies of Ap stars and binaries. On the other hand, we do not know how much the subclusters in an association differ from each other. We should look at all the characteristics (rotational velocities, frequencies of binaries, Ap and Am stars) of subclusters in a larger complex like the I Orion association; there is some evidence for such differences in the ScoCen association.

Slettebak: Your comment to the effect that clusters with large total mass tend to show larger $v$ $\sin i$ 's than clusters with small total mass is extremely interesting. Do you find a fairly strict correlation among the clusters thus far investigated?

$A b t:$ No, data are lacking on the lengths of main sequences in many clusters and associations, so we probably cannot tell at present. 
Collins: Do the data relating to $\langle v \sin i\rangle$ vs. spectral type and indicating the dip at B2 include Be stars? If not, would their inclusion remove the dip at B2?

$A b t$ : That sounds like a very reasonable suggestion. There seems to be no reason why the Be stars, which occur primarily in the vicinity of the B2 dip, should be excluded unlike, for example, the Am stars, whose rotational velocities may represent later modifications. On the other hand, there are nearly as many Be stars with types of $\mathrm{B} 3$ and $\mathrm{B} 5$ as of $\mathrm{B} 2$, so that including the Be stars does not remove the dip, unless the types of Be stars are often too late for their masses.

Van den Heuvel: Dr. Conti has shown that among the early A-stars there are stars similar to Am stars which, however, can only be recognized at high dispersion, and which therefore will mostly be classified as 'normal' at the dispersions which are employed in the work you referred to. Should not these be left out, or did you leave them out? The dip near A1 in the $\langle v \sin i\rangle$ curve for field stars might disappear in such a case.

$A b t$ : If one wishes to recover the original rotational velocities of stars, we should probably exclude the Sirius type or hot Am stars because their rotational velocities represent later modifications. But such stars have not been identified in the complete sample of Slettebak stars.

Deutsch: I think Conti has found that nearly enough of the sharp-line stars near A0 are abnormal, either in the sense of showing the Ap or 'hot Am' characteristic, to account for the excess number of sharp-line stars. These stars would also depress the value of $\langle v \sin i\rangle$.

Buscombe: (1) From preliminary estimates of rotational velocity for members of the Scorpio Centaurus Association, I showed (Irish Astron. J. $7(1965), 63)$ that the frequency distribution as a function of spectral type extends towards hotter spectral types the trend earlier established for II Per, which is a coeval association of the same space velocity stream. The very high incidence of duplicity among Sco-Cen stars must be taken into account. Moreno (Santiago Publ. 5 (1969), 72) has evidence of the correlation of narrow-band photometric indices on $v \sin i$ from Slettebak's more reliable individual determinations. The divergent ( $\langle v \sin i\rangle$, spectral class) distribution for members of NGC 6475 confirms my earlier assertions that this cluster is not a subgroup of the Sco-Cen association; NGC 6475 is more evolved and has a different space motion.

(2) Hyland (unpublished Ph.D. dissertation, Canberra, 1967) while investigating model atmospheres for the nine brightest stars (including Si-rich B peculiars) in each of two southern clusters, established that the relative frequency of fast rotators is high in IC 2602, but low in IC 2391.

(3) There is growing evidence that several sets of classification for B stars based on the precepts of Morgan and Keenan, show considerable influence of the use of slow or fast rotators among the standards.

$A b t$ : Regarding the third point, the originators of the MK system cannot be responsible for results obtained when their recommendations are not followed, especially with regard to the value of low dispersions $(\simeq 125 \AA / \mathrm{mm})$ and low resolution $(25-30 \mu$ ). Collins has shown that under those conditions the rotational effects on spectral classifications should be trivial, except for stars near the critical velocities. 